\title{
A science-based use of ensembles of opportunities for assessment and scenario studies
}

\author{
E. Solazzo and S. Galmarini \\ European Commission, Joint Research Centre, Institute for Environment and Sustainability, Air and Climate Unit, Ispra, Italy \\ Correspondence to: S. Galmarini (stefano.galmarini@jrc.ec.europa.eu)
}

Received: 30 September 2014 - Published in Atmos. Chem. Phys. Discuss.: 2 December 2014

Revised: 4 February 2015 - Accepted: 14 February 2015 - Published: 6 March 2015

\begin{abstract}
The multimodel ensemble exercise performed within the HTAP project context (Fiore et al., 2009) is used here as an example of how a pre-inspection, diagnosis and selection of an ensemble, can produce more reliable results. The procedure is contrasted with the often-used practice of simply averaging model simulations, assuming different models produce independent results, and using the diversity of simulation as an illusory estimate of model uncertainty. It is further and more importantly demonstrated how conclusions can drastically change when future emission scenarios are analysed using an un-inspected ensemble. The HTAP multimodel ensemble analysis is only taken as an example of a widespread and common practice in air quality modelling.
\end{abstract}

\section{Introduction}

A multimodel (MM) ensemble is defined as a group of simulations of the same case study, produced by formally different models, which are statistically treated in an attempt to improve the quality of the result (Potempski and Galmarini, 2009). Given the ever-increasing collaborations of geophysical modelling communities in joint assessment studies, MM ensembles are becoming very popular and an opportunity to extend and generalize individual deterministic model results (Solazzo et al., 2012 and 2013; Solazzo and Galmarini, 2014; Galmarini et al., 2004; Vautard et al., 2012; Evans et al., 2013; Bishop and Abramowitz, 2013; and many others).

In particular in atmospheric sciences, MM ensembles are used extensively in climate and air quality predictions and assessments. While in climate research and applications many of the concepts applied and described here are well known and correctly used, in air quality this is not always the case and several are the examples of direct use of un-inspected MM ensembles. We define an inspected MM ensemble (as opposed to an un-inspected one) as a set of model results whose properties and characteristics have been analysed in an attempt to reduce the presence of redundant information or elements that are not relevant to the determination of an accurate result. An inspected ensemble is expected to produce a result that is more accurate than the simple average of the MM results, at least in all the cases when the members of the ensemble are not independent (e.g. Kioutsioukis and Galmarini, 2014).

The motivations behind the necessity to inspect a MM ensemble are connected to the way in which MM ensembles are put together and to the nature of the participating models. In fact, the selection of the models whose results are "ensemble" is not, to the best of our knowledge and at least for air quality applications, regulated by any science-based criteria and there is no a priori specification that defines the characteristics of a model that should or should not be part of an ensemble. The constitution of a MM ensemble is merely based on an opportunity to provide model simulations and to participate in a community activity in which anyone is welcome (ensemble of opportunity). Regarding the nature of the models producing results for ensemble applications, one should never forget that the best results are those produced by ensembles of independent (and accurate) models (Potempski and Galmarini, 2009; Kioutsioukis and Galmarini, 2014; Weigel et al., 2008; Pirtle et al., 2010; Knutti, 2010; Knutti et al., 2010; Riccio et al., 2012). Formally, model $\mathrm{m}_{1}$ is defined as independent of $\mathrm{m}_{2}$ if the joint probability $p$ for a result of $\mathrm{m}_{1}$ and $\mathrm{m}_{2}$ can be expressed as $p\left(\mathrm{~m}_{1}, \mathrm{~m}_{2}\right)=p\left(\mathrm{~m}_{1}\right) p\left(\mathrm{~m}_{2}\right)$. When many independent models are combined together their bias can be randomly positive or negative, increasing the 
probability of cancelling out and of the sampled uncertainty not overlapping (Knutti et al., 2010; Abramowitz, 2010; Solazzo et al., 2013). Models used in air quality (among others) are not independent - they often share common assumptions, modules and input data. In most of the cases the models are different (phenotypical model difference; Potempski and Galmarini, 2009) but are not independent. This leads to the possibility that results obtained from an ensemble, rather than representing a true alternative and independent solution, would just be like in music composition a variation on the theme, producing a false sense of variability which could lead to coinciding (diverging) biased results and a false sense of agreement (uncertainty).

MM ensembles derived from simply different models are prone to redundancy and overconfidence. The inspection is therefore primarily finalized at

- the identification of the level of diversity (communality) shared by the model results

- retaining only those that are contributing with original information

- removing the redundancy.

Techniques exist which allow such screenings that rely on the existence of observations and the comparison of the ensemble variability with the observational variability (Potempski and Galmarini, 2009; Solazzo et al., 2013; Riccio et al., 2012).

In this study we aim to demonstrate the importance of using existing good practices in the air quality MM ensemble context. To that end we have selected a case study published in the past which does not exploit the true value of having multiple model results at hand. The case analysed is the HTAP (Hemispheric Transport of Air Pollution) phase 1 multimodel exercise (Dentener et al., 2010) and in particular the multimodel ensemble activity performed within it and presented by Fiore et al. (2009). The study of Fiore et al. (2009) is used here as merely representative of a widespread practice in the air quality modelling communities at all scales and it represents just an example of how things could be improved further. The MM ensemble by Fiore et al. (2009) is original in many aspects and, in particular, is used for sensitivity studies with respect to emission reduction options. The inspection of the ensemble can have important consequences also for emission scenarios as shown later, an aspect never considered before in the literature.

\section{The case study and MM ensemble inspection}

In 2006 the Task Force on Hemispheric Transport of Air Pollution (http://www.htap.org/) organized a comparison exercise of global and hemispheric transport models, focusing on the relationships between regional-scale emission perturbations and the response in air-quality, ecosystem- and climate- related variables. The information was used in an aggregated form to evaluate air pollution abatement strategies and their impact across the Northern Hemisphere. Results of the comparison exercise are summarized in Dentener et al. (2010), Sanderson et al. (2008), Fry et al. (2012), Wild et al. (2012), Jonson et al. (2010), Anenberg et al. (2009) and Fiore et al. (2009).

We focus on the MM ensemble analysis of Fiore et al. (2009) (henceforth FetA09). In FetA09, an average of 21 model results was used to investigate the monthly mean surface ozone concentration in three subregions of Europe (the Mediterranean, Central Europe with receptors between 0 and $1 \mathrm{~km}$ height, and Central Europe with receptors between 1 and $2 \mathrm{~km}$ height), five North American subregions (Northeast, Southwest, Southeast, Great Lakes, and Mountainous) and one Japanese subregion (EANET stations). Operational scores (bias, correlation coefficient and standard deviation) were calculated in each subregion making use of ground-based measurements. The combined spatial and temporal average of the modelled concentration values resulted in smoothed monthly time series. The analysis of FetA09 reveals that the distribution of the results is rather symmetric (Fig. 1). Supported by the agreement with observations, the authors considered the MM ensemble mean to be the best possible estimate as it "generally captures the observed seasonal cycle and is close to the observed regional mean" [FetA09], thus justifying the use of the MM ensemble mean to quantify source-receptor relationships as well as ozone concentration response to changes in the emissions scenarios.

The analysis by FetA09 was not aimed at proving the robustness of the MM ensemble mean, and provides an example of the widespread practice of averaging all available members, assuming that the average of many model results is always a better result than that of one model. That would be true if the models were independent but there is no a priori proof of that. Some questions arise: how robust are the results if the members are not independent models? How different would the result be should some model not taking part in the activity or more outliers (like the one in Fig. 1) be present? How generalized is the result since the selection of the ensemble members is based on the voluntary participation to a joint activity and the MM ensemble does not contain all possible results? Is there any duplication of information? Is all the information contained in a MM ensemble relevant and necessary? Since the construction of a MM ensemble is not governed by scientific selection criteria, the subsequent ensemble result strictly depends on aleatory factors and one can presume that it lacks generality as it is supported by assumptions known to be valid for independent members only.

The screening methodology we propose, and that we apply as an example to the FetA09 set, is a good way to exploit an abundance of model results in the best way, to transform the aleatory gathering of information into a more robust result that is based on general selection criteria. The large ensem- 

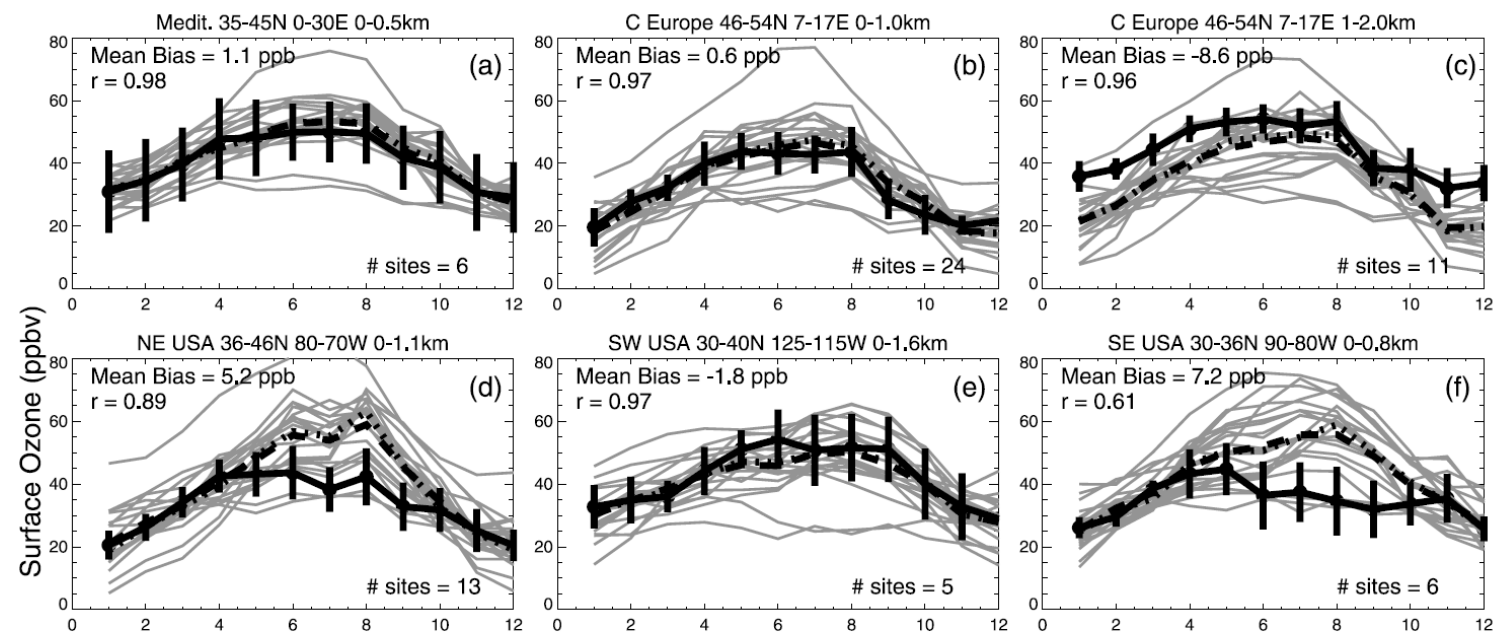

SW USA 30-40N 125-115W 0-1.6km
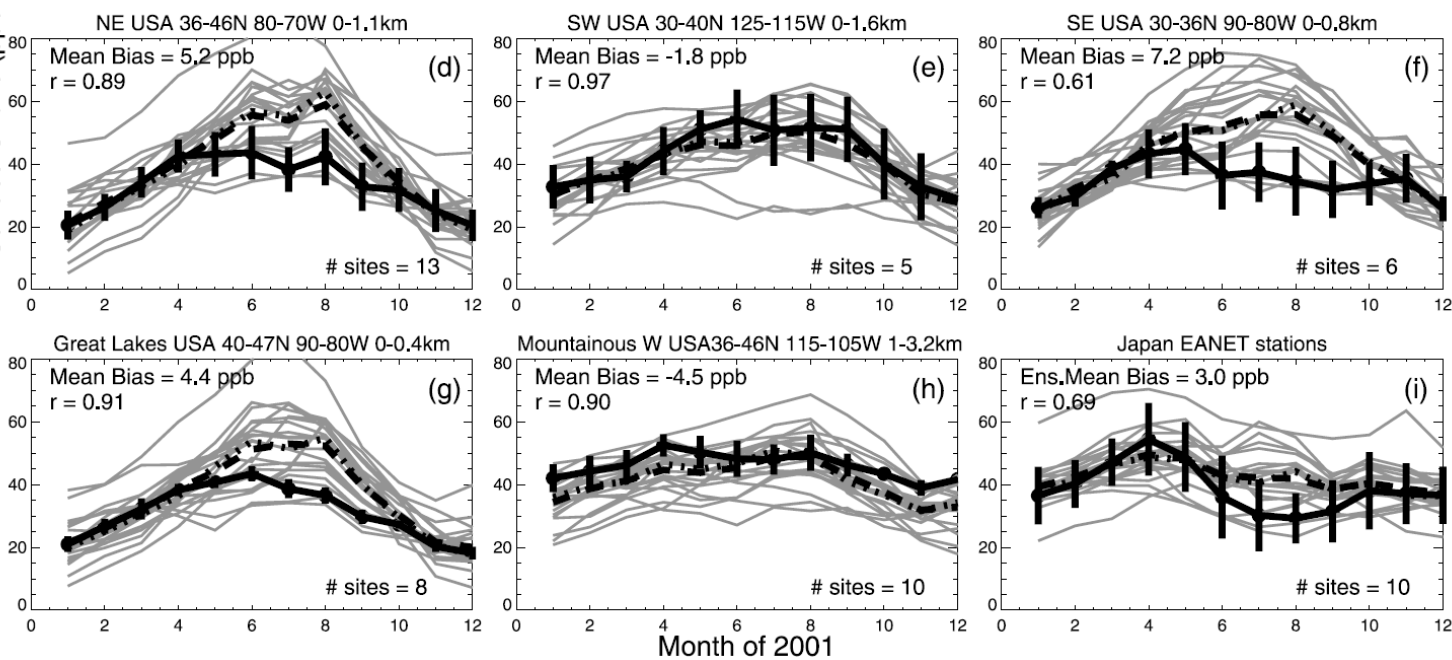

Figure 1. From Fiore et al. (2009): monthly mean surface $\mathrm{O}_{3}$ concentrations (ppb) for the year 2001. Observed values (black circles) represent the average of all sites falling within the given latitude, longitude, and altitude boundaries and denoted by the symbols in Fig. 1; vertical black lines depict the standard deviation across the sites. Monthly mean $\mathrm{O}_{3}$ in the surface layer of the SR1 simulations from the 21 models are first sampled at the model grid cells containing the observational sites and then averaged within subregions (grey lines); these spatial averages from each model are used to determine the multimodel ensemble median (black dotted line) and mean (black dashed line). Observations are from CASTNET (http://www.epa.gov/castnet/) in the United States, from EMEP (http://www.nilu.no/projects/ccc/emepdata.html) in Europe, and from EANET (http://www.eanet.cc/eanet.html) in Japan.

ble of model results becomes an opportunity to cherry-pick those models whose combination produces the most accurate $\mathrm{MM}$ ensemble and use only those to drive conclusions. The analysis will help to identify the size of the non-redundant ensemble and the subsets of members that produce skilled results.

\subsection{Inspecting a multimodel ensemble}

In this section the MM ensemble of FetA09 is inspected. We will concentrate on the ozone simulations over the same regions presented in FetA09 and we will make use of exactly the same model data and observations as used by FetA09, as the main point of the investigation here is to show that the results are different when an inspected MM ensemble is adopted. The inspection is based on the following steps:

- determine to what extent the variability (standard deviation about the ensemble mean as in Fortin et al., 2014) present in the observation is reproduced by the ensemble;
- determine the minimum number of models necessary to represent the observed variability;

- identification of the models forming the reduced MM ensemble used for subsequent analysis.

\subsubsection{The "accounted for" variability: eigenanalysis and ranked histogram technique}

The goal of this first analysis is to determine to what extent the observational variability is reproduced by the ensemble. An optimal situation is one in which the variability of observations coincides with that produced by the ensemble of models - in other words the ensemble of the results all together covers the same range of variation of the measurements. Any deviation from this condition, namely a smaller or a larger variability of the MM ensemble with respect to the observed one, would show, on one hand, the incapacity of the ensemble to span the observed reality, or on the other, the addition of irrelevant information to the simulation of the observed situation. Therefore, considering that a MM ensemble is assembled on an opportunity basis rather than results characteristics, this first step is of primary importance to es- 
timate to what extent the gathered set is appropriate for the case study.

A technique to assess the variability and to estimate the redundancy of the MM ensemble with respect to that of the observations was suggested by Annan and Hargreaves (2010) and applied in several MM ensemble modelling contexts (see e.g. Solazzo et al., 2013; Solazzo and Galmarini, 2014). It consists of projecting the observation anomalies (the element-wise difference between the observations and their mean) onto the principal components (PCs) of the covariance matrix of the deviation of the ensemble of models from the MM mean (the element-wise difference between each model realization and the MM ensemble mean). Principal component analysis (Jolliffe, 2002) is probably the most well-known and widespread dimension reduction technique. It is based on eigenanalysis to select uncorrelated directions associated with the largest variances.

When applied to the HTAP 21-member ensemble analysed by FetA09, this method shows that the first (largest) eigenvalue already explains more than $90 \%$ of the observational variability in most regions, the only exception being Japan with $60 \%$. In other words, most of the ensemble members have a significant projection onto the first eigenvector defining the major component, thus explaining the same portion of variance. If too many models are projected on the same eigenvector, it means that there are too many models producing repeating or "overlapping" solutions (thus, the MM ensemble is redundant and overconfident). A well-behaved MM ensemble (not necessarily the theoretical case of independent models) should be made of a number of models whose eigenvalues contribute to the explanation of as many different components as the observational variability and the ratio model-to-observed variance should be close to unity. In the case of the HTAP MM ensemble, when all eigenvalues are taken into account (and all of the associated eigenvectors), the MM ensemble variance is 4.7, 6.0, 8.7 times the variance of the observation anomalies for the EU Mediterranean, Central 0-1 km and Central 1-2 km regions respectively. Concerning the US Mountains, Great Lakes, SE, NE and SW regions, the full MM ensemble mean accounts for 25.4, 9.1, 20.6, 10.7 and 5.6 times the observed variability, respectively, and finally 4.7 times for the Japanese subregion. According to the definition of Annan and Hargreaves (2010) the ensemble is therefore wide, i.e. its variability is larger than the observed one. Dealing with a wide ensemble implies that there is a substantial amount of redundant variability, i.e. variability already accounted for by other models. Not all information contained in the ensemble is needed in principle and needs to be reduced.

An alternative method to diagnose the variability spanned by an ensemble of models to the eigenvalues used is the Talagrand or ranked histogram (RH) (Talagrand et al., 1998), which provides an evaluation of the consistency of the ensemble with an observed quantity. In a RH the observations are ranked in a number of bins equal to the number of mod- els making up the ensemble plus one for the extremes. The ensemble members are sorted to define ranges or "bins" of the modelled variable such that the probability of occurrence of the observation within each bin is, ideally, equal. The bins are determined by ranking the ensemble member from lowest to highest. The interval between each pair of ranked values forms a bin. An $N$-member ensemble corresponds to $N+1$ bins (Hamill, 2001). The underlying assumption is that each ensemble member in principle introduces an independent degree of variability. An indication of an ill-constructed ensemble is the ratio between the number of elements and the number of data available per model. If there are $N$ models with time series each of size $n_{\mathrm{t}}$ (elements of the time series), the implication of $N>n_{\mathrm{t}}$ is that there will be at least $N-n_{\mathrm{t}}$ empty bins in the RH, indicating redundancy of the ensemble and that the ensemble is inappropriate for the case analysed. This same result could be visualized by looking at the load factors resulting from the decomposition in PCs: many projections would be null, as the number of eigenvectors is larger than the number of data to project. For the HTAP MM ensemble used in this example, $N=21$ and $n_{\mathrm{t}}=12$. The RH for the nine subregions is reported in Fig. 2. Six (NA NE) to nine (NA SW) bins out of 22 are populated, (i.e. contain nonzero values), due to insufficient data and excess of redundant information. The use of the RH reveals another important problem with the FetA09 MM ensemble. Good ensemble practice would require $n_{\mathrm{t}} \gg N$. The plots clearly show that there are many empty bins (and therefore degrees of freedom in the process that are not part of the reality as no observations are present in that range). The uneven distribution of the histograms shows that much emphasis (overconfidence) is given to some aspects of the process description, while others are neglected - that is another way of representing the redundancy obtained with PC analysis presented earlier.

\subsubsection{Effective number of models}

Having assessed that the ensemble is redundant it is important to determine the minimum number of models from those available in the MM ensemble that would suffice to describe the observational variability. A method developed by Bretherton et al. (1999), and firstly applied to air quality models by Solazzo et al. (2013), quantifies the effective number of models sufficient to reproduce the variability of the observation as

$N_{\text {eff }}=\frac{\left(\sum_{k=1}^{N} \lambda_{k}\right)^{2}}{\sum_{k=1}^{N} \lambda_{k}^{2}}$

with $\lambda$ eigenvalue of the $\operatorname{corr}\left(d_{i}, d_{j}\right)$ matrix, which contains the linear correlation coefficient between any pair $d_{i}$, $d_{j}(i, j=1, \ldots, N)$, where $d$ is a metric defined according to Pennel and Reichler (2011):

$d_{\mathrm{m}}=e_{\mathrm{m}}-R \mathrm{MME}$, 

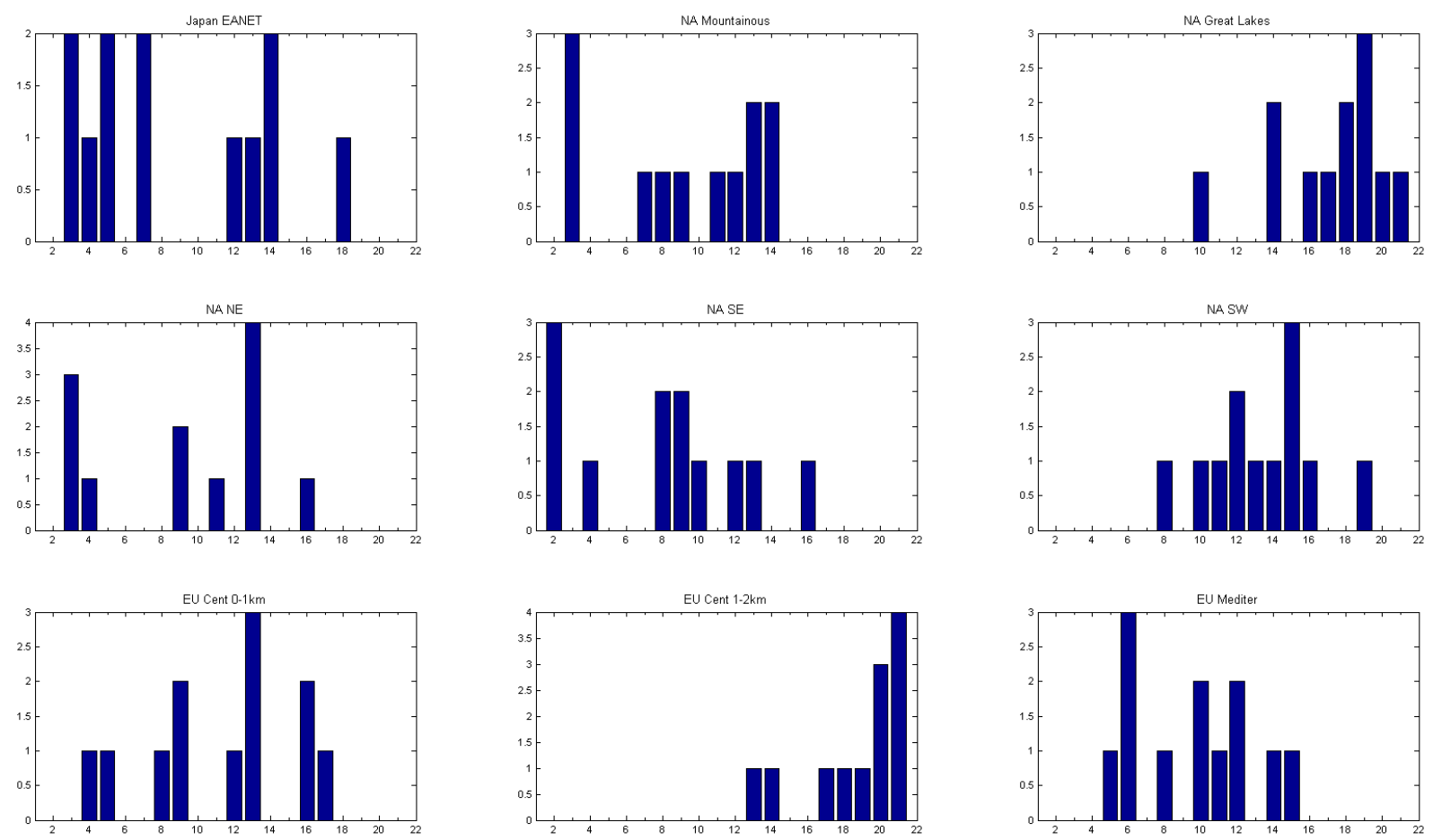

Figure 2. Ranked histogram for the nine subregions subject to MM ensemble evaluation.

where the index " $m$ " identifies the model, MME is the multimodel error (the average of all individual model's errors) and $R$ is the Pearson correlation coefficient between $e_{\mathrm{m}}$ (the error of model $\mathrm{m}$ ) and the MME. The removal of MME in Eq. (2) makes model errors more dissimilar from one another and uncovers "hidden" trends that are outweighed by overarching commonalities. Indeed, the scope of the metric $d_{\mathrm{m}}$ is to determine similarities between models beyond the dominating ones induced by shared inputs and/or common parametrizations to the extent that the former are accounted for in the average. The relationship (1) should be interpreted as: only if all eigenvalues were equal to unity, would Eq. (1) take a value of $N_{\text {eff }}=N$, which corresponds to the situation where all directions are equally important and all models add independent contributions to the explanation of the observational variability. On the other hand, if all error fields were similar, only one eigenvalue would be non-zero and $N_{\text {eff }}=1$. Equation (1) provides an analytical estimate of the dimensions of the subspace of models necessary to produce the information of the whole ensemble.

For the HTAP MM ensemble of FetA09, Eq. (1) gives $N_{\text {eff }}$ ranging between $\sim 2$ and 4 for the regions analysed by FetA09 compared to the original 21 models (Table 1). Thus, approximately three-quarters of the available members participate in the ensemble with already "accounted for" information. This is a revealing result which indicates paradigmatically the relevance of a pre-inspection of an ensemble. What seemed like a largely populated ensemble turns out to be incapable of capturing several degrees of freedom of ob- servations and 2-4 members of 21 are sufficient to describe the observational variability. One may ask: if so, why is the average of the 21 models fitting so well with the observations as presented in FetA09? The answers could be: pure chance, since finally the model results participated out of good will, and happened to be there in the right mixture. Just consider what would have happened to the mean of the models should one of the two most evident outliers in Fig. 1 decide to withdraw from the exercise. Alternatively an explanation could be the massive smoothing due to the monthly averaging along with the high level of tuning of the models around specific solutions that are normally distributed around the average observed data.

\subsubsection{Reducing ensembles}

As demonstrated in the previous sections, the HTAP MM ensemble is redundant and in particular 2-4 members are sufficient to represent the observational variability while the rest do not add any new information. Similarly, the extra elements are likely to deteriorate any evaluation metrics applied to the ensemble. At this point we know that the number of models that are necessary and sufficient is smaller than 21 but we do not know which combination of members for every grouping produces the optimal ensemble.

Given $N$ members, there are $G=N ! /[r !(N-r) !]$ possible groups of $r$ elements. A straightforward way to identify the optimal ensemble (optimal subset) and maximize the accuracy of the ensemble is to analyse all the $G$ combinations 

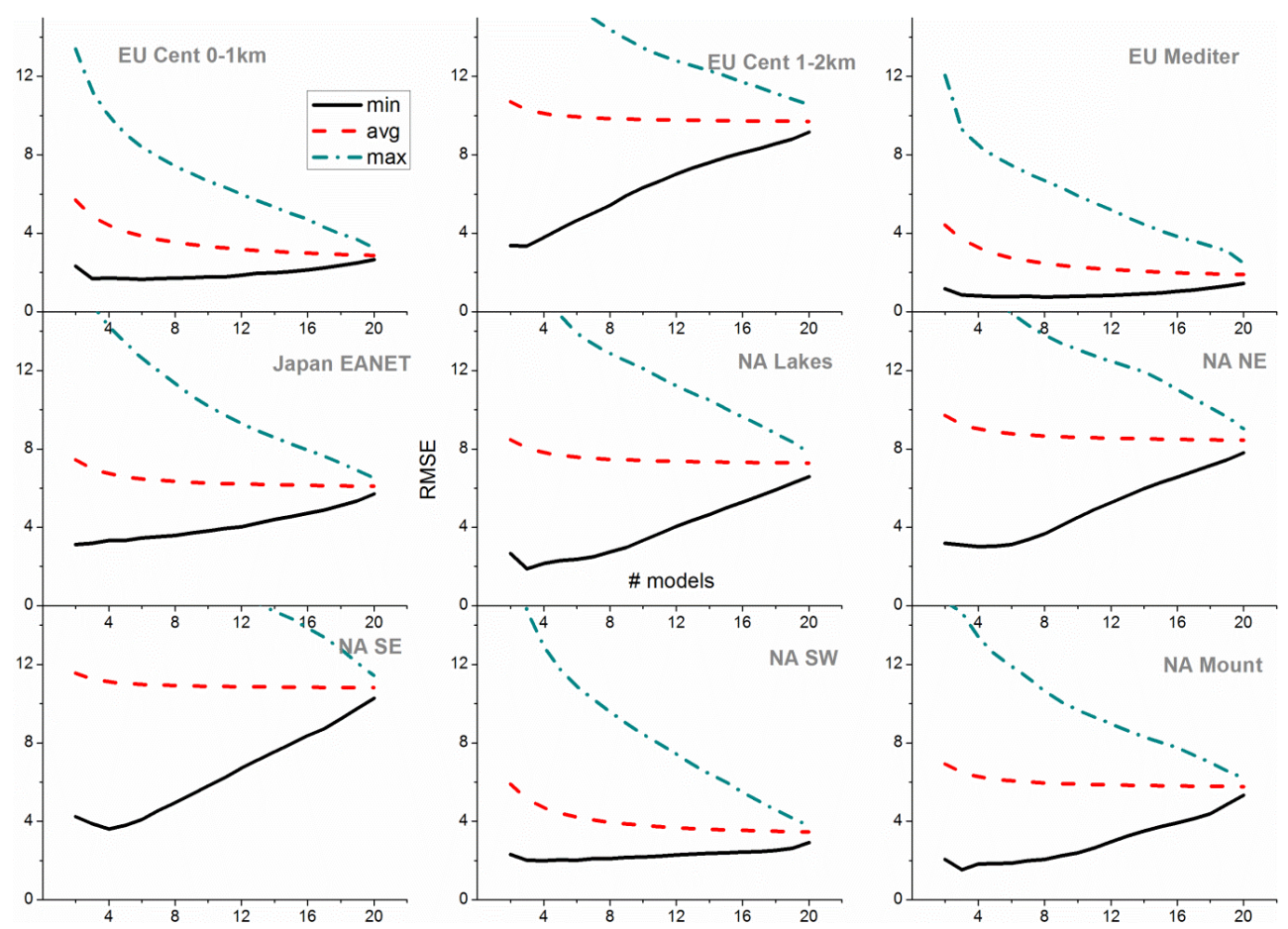

Figure 3. Maximum (dash-dotted), average (dashed), and minimum (continuous line) RMSE for all subsets of MM combinations and for the nine subregions subject to MM ensemble evaluation.

Table 1. Number of effective models $N_{\text {eff }}$ for the subregions object of the analysis - with reference to Fig. 2 of Fiore et al. (2009) top panel, based on $\operatorname{corr}\left(d_{i}, d_{j}\right)$. The $n_{\text {rec }}$ is the number of surface receptors used for evaluation.

\begin{tabular}{lc}
\hline Subregion & $N_{\text {eff }}$ \\
\hline EU Mediterranean region $\left(n_{\text {rec }}=6\right)$ & 4.0 \\
EU central region $0-1 \mathrm{~km}\left(n_{\text {rec }}=24\right)$ & 3.1 \\
EU central region $1-2 \mathrm{~km}\left(n_{\text {rec }}=11\right)$ & 3.5 \\
NE USA $\left(n_{\text {rec }}=13\right)$ & 1.9 \\
SW USA $\left(n_{\text {rec }}=5\right)$ & 1.8 \\
SE USA $\left(n_{\text {rec }}=6\right)$ & 1.9 \\
Great Lakes USA $\left(n_{\text {rec }}=8\right)$ & 2.0 \\
Mountainous USA $\left(n_{\text {rec }}=10\right)$ & 1.8 \\
Japan EANET $\left(n_{\text {rec }}=10\right)$ & 2.6 \\
\hline
\end{tabular}

of subsets of models and identify the one that minimizes the root mean square error (RMSE). The latter is a measure of the accuracy (the even distribution of model results from the observed value), and high accuracy also improves precision (a reduced spread/scatter of the model results around the observed value). In principle, measurement errors should be also taken into account in the procedure for reducing the ensemble, but in cases where they are significantly smaller than the model ones, the RMSE is sufficient measure.

In Fig. 3 we report the curves of minimum, mean and maximum RMSE for the nine subregions used by FetA09 as a function of the number of members of ensembles $(r=$ $2, \ldots, 21)$. The figure confirms the results on the number of models necessary to maximize the ensemble performance and tells us which combination of the 2-4 models out of 21 produces such improvement. The scores of the reduced ensemble are reported in Table 2 and are compared against the ones produced by the full ensemble mean. In all cases the mean of the reduced ensemble improves the accuracy (from $31 \%$ for NA NW to $71 \%$ for NA Mountain and NA Lakes) and precision (most notably for NA SE and NA NE). It can be seen that in several regions the use of the full MM ensemble of opportunity produces a clear deterioration in the ensemble statistics. In Table 2 we report also the ranking of the models contributing to minimize the error in the subregions. As can be seen from the table it is often the case that the error is minimized by a mixed rank (good performing and bad performing) group of members. In fact, if the two best models have a high chance of being also highly correlated then they would share some portion of information, thus resulting in some redundancy. Therefore when considering the ensemble mean of these two models, very little decrease in error would be found compared to the individual models. Mathematically, the theorems by Elashoff et al. (1967) and Cover (1974) have proven two important results on the selection of members and evaluation of individual scores: the best two models are seldom the combination of two models that maximizes the score of an ensemble average, and furthermore, the best single model may not appear in the ensemble max- 
Table 2. RMSE ranking and scores of the reduced MM ensemble mean for the subregions object of the analysis (RMSE: root mean square error; PCC: Pearson correlation coefficient; $\sigma$ : ratio of the modelled to the observed standard deviation).

\begin{tabular}{|c|c|c|}
\hline Domain & $\begin{array}{l}\text { Ranking of the } \\
\text { Min RMSE } \\
\text { combination }\end{array}$ & Score \\
\hline EU central 0-1 km & $1,15,19$ & $\begin{array}{l}\text { RMSE }=1.69(2.65) \\
\text { PCC }=0.98(0.96) \\
\sigma=0.99\end{array}$ \\
\hline EU central $1-2 \mathrm{~km}$ & $7,17,18$ & $\begin{array}{l}\text { RMSE }=3.35(9.2) \\
\text { PCC }=0.98(0.95) \\
\sigma=1.03\end{array}$ \\
\hline EU Medit & $4,6,13,15,19$ & $\begin{array}{l}\text { RMSE }=0.76(1.44) \\
\mathrm{PCC}=0.99(0.98) \\
\sigma=1.0\end{array}$ \\
\hline NA SW & $8,10,11,15$ & $\begin{array}{l}\text { RMSE }=2.0(2.9) \\
\text { PCC }=0.95(0.96) \\
\sigma=0.87\end{array}$ \\
\hline NA SE & $1,2,4,8$ & $\begin{array}{l}\text { RMSE }=3.61(10.27) \\
\text { PCC }=0.77(0.62) \\
\sigma=0.83\end{array}$ \\
\hline NA NE & $3,5,6,7$ & $\begin{array}{l}\mathrm{RMSE}=3.01(7.8) \\
\mathrm{PCC}=0.93(0.90) \\
\sigma=0.90\end{array}$ \\
\hline NA Mountain & $1,5,12$ & $\begin{array}{l}\text { RMSE }=1.53(5.33) \\
\mathrm{PCC}=0.93(0.90) \\
\sigma=1.04\end{array}$ \\
\hline NA Lakes & $1,5,6$ & $\begin{array}{l}\text { RMSE }=1.89(6.58) \\
\mathrm{PCC}=0.97(0.91) \\
\sigma=1.03\end{array}$ \\
\hline Japan EANET & 12,15 & $\begin{array}{l}\text { RMSE }=3.11(5.70) \\
\mathrm{PCC}=0.96(0.79) \\
\sigma=0.66\end{array}$ \\
\hline
\end{tabular}

imizing the feature score. As a result, the simple method of making ranked combinations of models with the best individual features may prove unsuccessful, as also demonstrated by e.g. Solazzo et al. (2013), Hannan and Hargreaves (2011), Kioutsioukis and Galmarini (2014), Knutti et al. (2010) and others. This confirms the importance of the inspection of the available results prior to their use and of having at disposal a large pool of models from which optimal subsets can be extracted.

\section{Impact on the results of emission sensitivity analysis of an inspected vs. uninspected ensemble}

An important part of FetA09 relates to the sensitivity study on emission reduction. As part of the HTAP programme the consequences of an emission reduction of $20 \%$ anthropogenic $\mathrm{NO}_{\mathrm{x}}$ in a specific part of the globe were investigated using the MM ensemble available. Since we have demonstrated that the MM ensemble used in FetA09 is redundant and having identified the optimal number of elements and the most accurate set of models, one may wonder how the predicted consequences of the emission reduction on ozone concentration would change if we used the reduced ensemble.

We focused the analysis on the North American region only. In FetA09 the use of the mean of the full ensemble produced an average response in ozone concentration of $-0.76 \mathrm{ppb}$ in the NA region as a consequence of the reduction of $\mathrm{NO}_{\mathrm{x}}$ emission by $20 \%$. Note that the NA region is subjected to the emission reduction and therefore the investigation includes the whole of the USA and part of Mexico (Fig. 1 of FetA09), and thus it has a spatial extension that includes the five NA subregions described in Sect. 2 for the evaluation. Furthermore, of the 21 models participating to the 
evaluation part of the exercise, only 14 model results were made available for the simulation with reduced emission scenarios. Therefore, for the sake of consistency, we repeated the redundancy inspection for the 14-member ensemble and calculated the most accurate set through the minimization of RMSE as described in Sect. 2.1.3. The size of the newly calculated subsets ranges between 3 for the Lakes, Northeast, Southwest and Southeast of the USA, and 4 for the Mountainous region. The newly calculated set obtained from the original 14-member ensemble produced an ozone concentration reduction of $2.32 \mathrm{ppb}$ on average across all regions, which is $300 \%$ more than that found by FetA09. The largest variation is obtained for the Southeast region of the USA, with an ozone concentration decrease of $5.30 \mathrm{ppb}$, which is fivefold that obtained by FetA09. Such an analysis demonstrates how conclusions can change if the ensemble is not inspected a priori and reduced if necessary.

In the exploration of scenario or sensitivity for ideal conditions like that presented in HTAP, one may be tempted to construct an ensemble that only groups the best-preforming model results in the evaluation against measurements, using only those in the sensitivity or scenario case study, grouping them in an ensemble. This would be wrong in principle or in other words would not produce the best ensemble by definition, as demonstrated by the already cited theorems of Elashoff et al. (1967) and Cover (1974).

\section{Conclusions}

Use of the multimodel ensemble is becoming very popular in geophysical studies. In this paper we have contrasted the results from an ensemble of opportunity casually assembled model of phenotypically different driving elements, with the results obtained for when the same pool of models is screened to eliminate redundancy and the optimal combination is used.

The case of HTAP phase 1 is taken here as an example of a practice that is widespread, especially in the realm of air quality, for atmospheric dispersion at all scales. A very limited amount of studies correctly apply the technique. The HTAP case has been selected for two main reasons:

- the very large number of models that participated in the initiative and that were available for the ensemble analysis;

- the ensemble results were also used as the basis to assess the consequences of an emission reduction strategy on ozone in several regions of the world.

The HTAP ensemble has been assessed against available measurements and the following conclusion were obtained:

- In spite of the large number of participating models, the scarcity of time steps produces an important level of redundancy as seen from the simple analysis of a ranked histogram.
- A smaller subset of models performs much better when compared to measurements and it is statistically more significant.

- In the case of HTAP [FetA09] the objective of the study was to determine, through a multi-model ensemble, the impact of emission changes produced in one continent on another. The analysis conducted on the impact over the same continent where the emissions are produced, reveals that the conclusions remain the same as those produced by FetA09 but the values found are between 3 and 5 times higher when using a non-redundant ensemble.

These are problems that are common to many multi model studies and for which a minimum set of good practice rules should be taken into account (Kioutsioukis and Galmarini, 2014). Among these, we point out that in order to have any reasonable statistics the number of measurements should be much greater than the number of ensemble members. Otherwise the rank histogram is simply not a proper tool for the analysis.

On a more general level, it is clear that the use of uninspected ensembles of opportunities is a mispractice that could lead to under-exploitation of the latter and in some case even wrong conclusions. Quantitative practices guarantee the best possible diagnosis of the ensemble potential and its full exploitation. The availability of monitoring information is essential for the performance of the analysis presented here and it could be argued that the optimal ensemble identification is prone to the time and spatial representativity of the observations. This is true for the evaluation of any individual model result that depends on the space and time distribution of observation and the phenomenology represented.

The hemispheric transport case analysed here also raises the issue of the space- and timescale in which a set of models verified in a certain area could be used. Verification of the effect that an optimal set of an ensemble, based on data pertaining to a specific region and time frame, has in another region remains an important element of research - whether, in other words, an optimal set selected for region $A$ using observations in region $A$ can be used for a region $B$ and in a scenario or sensitivity analysis mode. Scale dependence of the atmospheric processes involved could become an issue in this case, and will have to be verified. On the other hand we consider the use of the optimal set for scenario and sensitivity study in the area where the observations used for its selection have been collected much more appropriate than the use of a full ensemble of opportunity. The selection of the optimal set through observations on a base case scenario is equivalent to the evolution of a single deterministic model and its application for speculative scenario analysis or forecast applications.

The representativity of the multi-model ensemble compared to observation and the minimization of redundancy remain important issues. In the light of what we specu- 
late here, the use of multiscale multimodel ensembles, constructed with combinations of models covering different portions of the atmospheric power spectrum, could greatly improve representativity and provide coverage of the problem in a much more detailed form. The combination of globaland regional-scale results, for example, in one ensemble is a possibility that will be explored in the framework of the next phase of HTAP.

Acknowledgements. Arlene Fiore (Columbia University) and the HTAP modelling community are acknowledged for making the model and observational data available for the current analysis (http://www.htap.org/) and for their openness to our investigation. Frank Dentener (JRC) is acknowledged for the valuable comments that greatly improved this paper. The authors also thank Brigitte Koffi (JRC) for having retrieved some of the data used in this paper.

Edited by: J. Brandt

Reviewed by: S. Potempski and two anonymous referees

\section{References}

Abramowitz, G.: Model independence in multi-model ensemble prediction, Australian Meteorological and Oceanographic Journal, 59, 3-6, 2010.

Anenberg, S. C., West, J. J., Fiore, A. M., Jaffe, D. A., Prather, M. J., Bergmann, D., Cuvelier, K., Dentener, F. J., Duncan, B. N., Gauss, M., Hess, P., Eiof Jonson, J., Lupu, A., MacKenzie, I. A., Marmer, E., Park, R. J., Sanderson, M. G., Schultz, M., Shindell, D. T., Szopa, S., Garcia Vivanco, M., Wild, O., and Zeng, G.: Intercontinental Impacts of Ozone Pollution on Human Mortality, Environ. Sci. Technol., 43, 6482-6487, 2009.

Annan, J. D. and Hargreaves, J. C.: Reliability of the CMIP3 ensemble, Geophys. Res. Lett., 37, L02703, doi:10.1029/2009GL041994, 2010.

Bishop, C. H. and Abramowitz, G.: Climate model dependence and the replicate Earth paradigm, Clim. Dynam., 41, 885-900, 2013.

Bretherton, C. S., Widmann, M., Dymnikov, V. P., Wallace, J. M., and Bladè, I.: The effective number of spatial degrees of freedom of a time-varying field, J. Climate, 12, 1990-2009, 1999.

Cover, T. T.: The best two independent measures are not the two best, IEEE Trans. System Man. Cybernetics, 4, 116-117, 1974.

Dentener, F., Keating, T., and Akimoto, H. (Eds.): Hemispheric Transport of Airpollution, Part A, Ozone and Particulate Matter, in: Economic Commission for Europe, Air Pollution Studies, 17, ISBN 978-92-1-117043-6, UNECE, Geneva, 2010.

Elashoff, J. D., Elashoff, R. M., and Goldman, G. E.: On the choice of variables in classification problems with dichotomous variables, Biometrika, 54, 668-670, 1967.

Evans, J. P., Ji, F., Abramowitz, G., and Ekstrom, M.: Optimally choosing small ensemble members to produce robust climate simulations, Environ. Res. Lett., 8, 044050, doi:10.1088/17489326/8/4/044050, 2013.

Fiore, A. M., Dentener, F. J., wild, O., Cuvelier, C., Schultz, M. G., Hess, P., Textor, C., Schulz, M., Doherty, R. M., Horowitz, L. W., MacKenzie, I. A., Sanderson, M. G., Shindell, D. T., Stevenson, D. S., Szopa, S., Van Dingenen, R., Zeng, G., Atherton, C.,
Bergmann, D., Bey, I., Carmichael, G., Collins, W. J., Duncan, B. N., Faluvegi, G., Folberth, G., Gauss, M., Gong, S., Hauglustaine, D., Holloway, T., Isaksen, I. S. A., Jacob, D. J., Jonson, J. E., Kaminski, J. W., Keating, T. J., Lupu, A., Marmer, E., Montanaro, V., Park, R. J., Pitari, G., Pringle, K. J., Pyle, J. A., Schroeder, S., Vivanco, M. G., Wind, P., Wojcik, G., Wu, S., and Zuber, A.: Multimodel estimates of intercontinental sourcereceptor relationships for ozone pollution, J. Geophys. Res., 114, D04301, doi:10.1029/2008JD010816, 2009.

Fortin, V., Abaza, M., Anctil, F., and Turcotte, R.: Why should ensemble spread match the RMSE of the ensemble mean, J. Hydrometeor., 15, 1708-1713, 2014.

Fry, M. M., Naik, V., West, J. J., Schwarzkopf, M. D., Fiore, A. M., Collins, W. J., Dentener, F. J., Shindell, D. T., Atherton, C., Bergmann, D., Duncan, B. N., Hess, P., MacKenzie, I. A., Marmer, E., Schultz, M. G., Szopa, S., Wild, O., and Zeng, G.: The influence of ozone precursor emissions from four world regions on tropospheric composition and radiative climate forcing, J. Geophys. Res., 117, D07306, doi:10.1029/2011JD017134, 2012.

Galmarini, S., Bianconi, R., Klug, W., Mikkelsen, T., Addis, R., Andronopoulos, S., Astrup, P., Baklanov, A., Bartniki, J., Bartzis, J. C., Bellasio, R., Bompay, F., Buckley, R., Bouzom, M., Champion, H., D’Amours, R., Davakis, E., Eleveld, H., Geertsema, G. T., Glaab, H., Kollax, M., Ilvonen, M., Manning, A., Pechinger, U., Persson, C., Polreich, E., Potemski, S., Prodanova, M., Saltbones, J., Slaper, H., Sofiev, M. A., Syrakov, D., Sørensen, J. H., Van der Auwera, L., Valkama, I., and Zelazny, R.: Ensemble dispersion forecasting - Part I: concept, approach and indicators, Atmos. Environ., 38, 4619-4632, 2004.

Hamill, T. M.: Interpretation of rank histograms for verifying ensemble forecasts, Mon. Weather Rev., 129, 550-560, 2001.

Jolliffe, I.: Principal component analysis, Springer, 2nd edition, 2002.

Jonson, J. E., Stohl, A., Fiore, A. M., Hess, P., Szopa, S., Wild, O., Zeng, G., Dentener, F. J., Lupu, A., Schultz, M. G., Duncan, B. N., Sudo, K., Wind, P., Schulz, M., Marmer, E., Cuvelier, C., Keating, T., Zuber, A., Valdebenito, A., Dorokhov, V., De Backer, H., Davies, J., Chen, G. H., Johnson, B., Tarasick, D. W., Stübi, R., Newchurch, M.J., von der Gathen, P., Steinbrecht, W., and Claude, H.: A multi-model analysis of vertical ozone profiles, Atmos. Chem. Phys., 10, 5759-5783, doi:10.5194/acp-10-57592010, 2010.

Kioutsioukis, I. and Galmarini, S.: De praeceptis ferendis: good practice in multi-model ensembles, Atmos. Chem. Phys., 14, 11791-11815, doi:10.5194/acp-14-11791-2014, 2014.

Knutti, R.: The end of model democracy?, Climate Change, 102, 395-404, 2010.

Knutti, R., Furrer, R., Tebaldi, C., Cermak, J., and Meehl, G. A.: Challenges in combining projections from multiple climate models, American Meteorological Society, 23, 2739-2758, 2010.

Pennel, C. and Reichler, T.: On the effective numbers of climate models J. Climate, 24, 2358-2367, 2011.

Pirtle, Z., Meyer, R., and Hamilton, A.: What does it mean when climate models agree? A case for assessing independence among general circulation models, Environ. Sci. Policy, 799, 351-361, 2010 . 
Potempski, S. and Galmarini, S.: Est modus in rebus: analytical properties of multi-model ensembles, Atmos. Chem. Phys., 9, 9471-9489, doi:10.5194/acp-9-9471-2009, 2009.

Riccio, A., Ciaramella, A., Giunta, G., Galmarini, S., Solazzo, E., and Potempski, S.: On the systematic reduction of data complexity in multi-model ensemble atmospheric dispersion modelling, J. Geophys. Res., 117, D05314, doi:10.1029/2011JD016503, 2012.

Sanderson, M. G., Dentener, F. J., Fiore, A. M., Cuvelier, C., Keating, T. J., Zuber, A., Atherton, C. S., Bergmann, D. J., Diehl, T., Doherty, R. M., Duncan, B. N., Hess, P., Horowitz, L. W., Jacob, D., Jonson, J.-E., Kaminski, J. W., Lupu, A., Mackenzie, I. A., Marmer, E., Montanaro, V., Park, R., Pitari, G., Prather, M. J., Pringle, K. J., Schroeder, S., Schultz, M. G., Shindell, D. T., Szopa, S., Wild, O., and Wind, P.: A multi-model source-receptor study of the hemispheric transport and deposition of oxidised nitrogen, Geophys. Res. Lett., 35, L17815, doi:10.1029/2008GL035389, 2008.

Solazzo, E. and Galmarini, S.: The Fukushima- ${ }^{137}$ Cs deposition case study: properties of the multi-model ensemble, J. Environ. Radioact., 139, 226-233, doi:10.1016/j.jenvrad.2014.02.017, 2014.

Solazzo, E., Bianconi, R., Vautard, R., Appel, K. W., Moran, M. D., Hogrefe, C., Bessagnet, B., Brandt, J., Christensen, J. H., Chemel, C., Coll, I., van der Gon, H. D., Ferreira, J., Forkel, R., Francis, X. V., Grell, G., Grossi, P., Hansen, A. B., Jericevic, A., Kraljevic, L., Miranda, A. I., Nopmongcol, U., Pirovano, G., Prank, M., Riccio, A., Sartelet, K. N., Schaap, M., Silver, J. D., Sokhi, R. S., Vira, J., Werhahn, J., Wolke, R., Yarwood, G., Zhang, J., Rao, S. T., and Galmarini, S.: Ensemble modelling of surface level ozone in Europe and North America in the context of AQMEI, Atmos. Environ., 53, 60-74, 2012.
Solazzo, E., Riccio, A., Kioutsioukis, I., and Galmarini, S.: Pauci ex tanto numero: reduce redundancy in multi-model ensembles, Atmos. Chem. Phys., 13, 8315-8333, doi:10.5194/acp-13-83152013, 2013.

Talagrand, O., Vautard, R., and Strauss, B.: Evaluation of probabilistic prediction systems, paper presented at aa seminar on predictability, Eur. Cent. For Medium Weather Forecasting, Reading, UK, 1998.

Vautard, R., Moran, M. D., Solazzo, E., Gilliam, R. C., Matthias, V., Bianconi, R., Chemel, C., Ferreira, J., Geyer, B., Hansen, A. B., Jericevic, A., Prank, M., Segers, A., Silver, J. D., Werhahn, J., Wolke, R., Rao, S. T., and Galmarini, S.: Evaluation of the meteorological forcing used for AQMEII air quality simulations, Atmos. Environ., 53, 15-37, 2012.

Weigel, A. P., Liniger, M. A., and Appenzeller, C.: Can multi-model combination really enhance skill of probabilistic ensemble forecast?, Q. J. Roy. Meteorol. Soc., 134, 241-260, 2008.

Wild, O., Fiore, A. M., Shindell, D. T., Doherty, R. M., Collins, W. J., Dentener, F. J., Schultz, M. G., Gong, S., MacKenzie, I. A., Zeng, G., Hess, P., Duncan, B. N., Bergmann, D. J., Szopa, S., Jonson, J. E., Keating, T. J., and Zuber, A.: Modelling future changes in surface ozone: a parameterized approach, Atmos. Chem. Phys., 12, 2037-2054, doi:10.5194/acp-12-20372012, 2012. 